Submitted to Ultrasonics

\title{
SURVEY OF ADVANCED NUCLEAR TECHNOLOGIES FOR POTENTIAL APPLICATIONS OF SONOPROCESSING
}

\author{
Floren Rubio', Edward D. Blandford ${ }^{1 *}$, and Leonard J. Bond ${ }^{2}$ \\ ${ }^{1}$ Department of Nuclear Engineering, University of New Mexico \\ MSC01 1120, 1 University of New Mexico Albuquerque, NM 87131 \\ ${ }^{2}$ Center for Nondestructive Evaluation, \& Department of Aerospace Engineering and Department \\ of Mechanical Engineering, Iowa State University, \\ 115A ASC II, 1915 Scholl Road, Ames, IA 50011
}

*Corresponding Author:

Edward D. Blandford,

Assistant Professor,

Department of Nuclear Engineering,

University of New Mexico,

MSC01 1120; 259 Farris Engineering Center

Albuquerque, NM 87131

TEL: +1 505-277- 7964

FAX: +1 505-277-5433

edb@unm.edu 
April, 2016

Professor, Arthur Every

Editor

School of Physics, University of the Witwatersrand, South Africa

Dear Prof. Arthur Every,

Please consider our manuscript entitled "SURVEY OF ADVANCED NUCLEAR TECHOLOGIES FOR POTENTIAL APPLICATION OF SONOPROCESSING” for publication in Ultrasonics.

Sincerely Yours,

Edward Blandford,

Assistant Professor, Department of Nuclear Engineering, University of New Mexico, MSC01 1120; 259 Farris Engineering Center, Albuquerque, NM 87101 TEL: +1 505-277- 7964

FAX: +1 505-277-5433 edb@unm.edu 


\author{
Ultrasonics \\ Manuscript Draft
}

\title{
Manuscript Number \\ Title: SURVEY OF ADVANCED NUCLEAR TECHNOLOGIES FOR POTENTIAL APPLICATIONS OF SONOPROCESSING
}

\author{
Article Type: Review Article
}

\begin{abstract}
:
Ultrasonics has been used in many industrial applications for both sensing at low power and processing at higher power. Generally, the high power applications fall within the categories of liquid stream degassing, impurity separation, and sonochemical enhancement of chemical processes. Examples of such industrial applications include metal production, food processing, chemical production, and pharmaceutical production. There are many nuclear process streams that have similar physical and chemical processes to those applications listed above. These nuclear processes could potentially benefit from the use of high-power ultrasonics. There are also potential benefits to applying these techniques in advanced nuclear fuel cycle processes, and these benefits have not been fully investigated. Currently the dominant use of ultrasonic technology in the nuclear industry has been using low power ultrasonics for non-destructive testing/evaluation (NDT/NDE), where it is primarily used for inspections and for characterizing material degradation. Because there has been very little consideration given to how sonoprocessing can potentially improve efficiency and add value to important process streams throughout the nuclear fuel cycle, there are numerous opportunities for improvement in current and future nuclear technologies. In this paper, the relevant fundamental theory underlying sonoprocessing is highlighted, and some potential applications to advanced nuclear technologies throughout the nuclear fuel cycle are discussed.
\end{abstract}

\section{HIGHLIGHTS:}

The application of power ultrasonics to nuclear processes is proposed.

Identified specific processes in the nuclear fuel cycle that can benefit from power ultrasonics.

The use of ultrasonics in the development of advanced nuclear technologies is proposed.

Material challenges for deploying ultrasonics in a nuclear process environment are discussed.

KEYWORDS:

sonoprocessing, sonochemistry, sparging enhancement, nuclear applications

\section{INTRODUCTION}

Sonoprocessing involves the use of ultrasound to deliver energy for producing physical or chemical effects at both laboratory scale and in a medium to be found in an industrial process stream. Sonoprocessing is generally divided between sonomechanical and sonochemical applications [1]. Sonomechanical applications utilize the agitation induced in the medium by sonic energy, and sonochemical applications, in general, utilize cavitation effects to enhance or catalyze a targeted chemical reaction. An example of sonomechanical processing is degassing of molten metals [2]. There are many sonochemical applications including those used in food processing, chemical manufacturing, and pharmaceutical production $[3,4]$.

Currently in the nuclear industry, the most common use of ultrasound is non-destructive testing/evaluation (NDT/NDE), where it is used for inspections at the time of construction and for periodic inspections that seek to detect and characterize significant material degradation [5]. There have also been a limited number of investigations that consider how sonoprocessing can potentially improve efficiency in interactions and add value to important process 
streams throughout the nuclear fuel cycle [6]. In this paper, we review and discuss the potential uses for sonoprocessing to process streams that are found in advanced nuclear technology throughout the nuclear fuel cycle.

A process stream can be defined as a fluid, or slurry, which undergoes some process or reaction using either batch or steady state flow through reactor. There are three general ways in which ultrasonic technology has been used to impart ultrasonic energy in to a process stream. The first of these is by direct immersion of a horn into the process fluid. The second is by attaching a transducer to the surface of the reactor pipe, and the third is by indirect coupling of the energy from a transducer through an intermediate fluid into the process stream. All three of these methods of imparting ultrasonic energy can be used to deliver higher power ultrasonic energy. Examples of basic designs for reactors which illustrate these approaches are shown in Figures 1-3 [7]. The design illustrated in Figure 2 would be difficult to implement in a cylindrical geometry due to the difficulty of producing and powering cylindrical transducers. In order to get around that constraint, transducers have been mounted in hexagonal or pentagonal configurations directed at the active region of pipe.

The efficiency and effectiveness of the various options for industrial sonoprocessing systems have been considered by several researchers including Thompson and Doraiswamy [7] and Gogate et al [8]. In sonoprocessing there are in general two general classes of interaction which are usually classified in terms of the nature of the resulting interaction process and the intensity of the ultrasonic energy employed. The simplest classification is that by dividing processes into those interactions which are above the cavitation threshold and those where cavitation does not occur and where energy is delivered to induce phenomena, such as streaming.

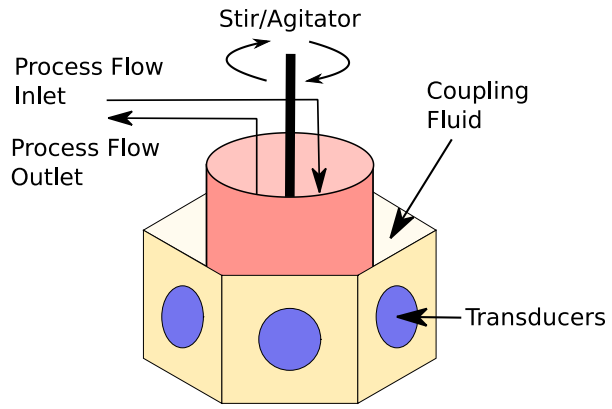

Figure 1. Implementation of indirect sonication is using a coupling fluid jacket around a cylindrical reaction volume [7]. ( 2 column fitting image)

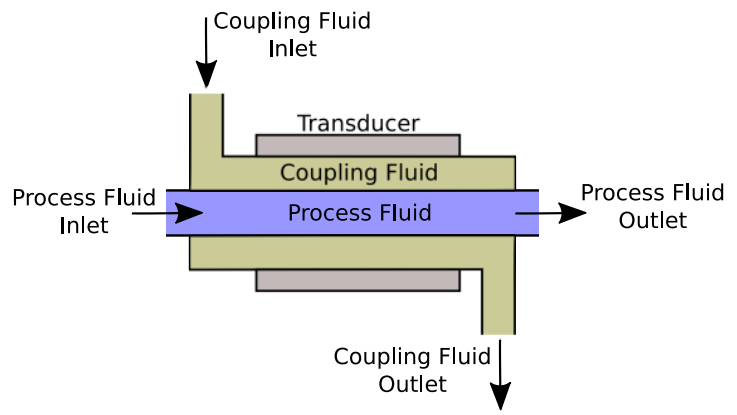

Figure 2. Process stream flowing through a coupling fluid jacket. [7]. (2 column fitting image) 


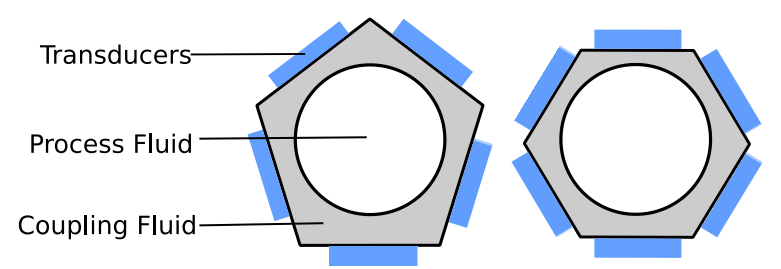

Figure 3. Cross sections of the ultrasonic coupling fluid jackets [7]. (2 column fitting image)

Ultrasonics generally is used to increase yield or enable a chemical reaction, by delivering energy, or provide a method of separation, such as degassing two constituents. In looking at potential new applications for sonoprocessing there are several challenges to consider when investigating the potential use of power ultrasonics to nuclear fuel-cycle process streams. First there is scale-up when moving from a prototypical pilot plant to a fully integrated industrial process. This is an issue for any proposed chemical reactor design and can, in many ways, be approached in a similar manner to non-nuclear applications. Second, the use of sonoprocessing for nuclear applications typically involves process streams operating under harsh conditions oftentimes including high temperature and pressure, as well as challenging radiation fields, and a more stringent regulatory environment, than is found for non-nuclear chemical processes. Finally, one must factor in the economic performance of the sonoprocessing technology where often times power ultrasonics involves a significant energy cost. Historically, the use of sonoprocessing has in general only been justifiable when there is a high value product being produced, such as in the pharmaceutical industry. Other industries that have been looking to increase the use of ultrasonics have included petro-chemical processing. A recent feasibility study has shown that implementing ultrasonic technology in the heavy oil industry gave good scale up economics and performance for their application [9]. In looking at potential applications in the nuclear industry it will be necessary to see where similar tradeoffs exist, and where the price point for implementing ultrasonics can be seen to be similarly favorable [9].

\section{BACKGROUND THEORY OF ULTRASONICS FOR ADVANCED NUCLEAR SONOPROCESSING APPLICATIONS}

Most of the demonstrated non-medical applications of sonoprocessing processing with high power ultrasonics take advantage of sonically induced cavitation $[1,10]$. The bubble formation and related energy exchange are transient, involve high pressures and temperatures. The behavior is nonlinear by nature and challenging to describe, particularly when multi-phase (slurry or catalytic particles) and multi-bubble phenomena occur $[1,7,11-13]$. Ultrasonics is in essence a tool that can be used to deliver energy into a medium. This sonic energy can then be manifested in interactions as vibrations and mechanical motion (acoustic streaming), heat (through rapid bubble compression and absorption), and light (sonoluminescence) all through cavitation and the related absorption and dissipation of the sonic energy. There are many factors that affect the sonically induced phenomena within a fluid, including temperature, pressure and fluid thermophysical properties. In order to comprehensively describe this behavior, the whole spectrum of phenomena must be considered ranging from interactions in single-phase fluid with relatively low intensity ultrasound to multi-phase slurry with high ultrasound intensity systems. There is also a further complication due to the spatial variations in the phenomena that occur at varying distances from the sonic source within a reactor, and all the complexities associated with variations caused by properties such a bubble density. In this paper a brief discussion of key topics is included.

\subsection{Cavitation}

Ultrasonic cavitation is generated during the rarefaction part of the period of a sound wave where the lowest pressures are induced. This phenomenon has been investigated and documented extensively [11-14]. The threshold for cavitation is a fundamental boundary in any process application and cavitation thresholds are affected by many factors. The most important factors are the static pressure and temperature (as it controls fluid properties), the presence of dissolved gas and/or particulate matter, fluid viscosity, the frequency of the applied ultrasound, and the wave induced pressure variation [14]. When gas and/or particulates are present in a fluid, these contaminants introduce "weak points" in the fluid, which make bubble formation in the fluid easier, and results in a lowering of the cavitation threshold. Viscosity describes the cohesive forces in a fluid and, in general, the transient acoustic pressure needed to 
cause cavitation varies directly with viscosity. When considering the effect of temperature, the pressure required for cavitation is inversely proportional to temperature. When the applied ultrasonic fields are considered, the intensity at which cavitation occurs is dependent on the frequency, and at higher frequencies it takes greater plane wave intensities to induce cavitation $[1,14,15]$.

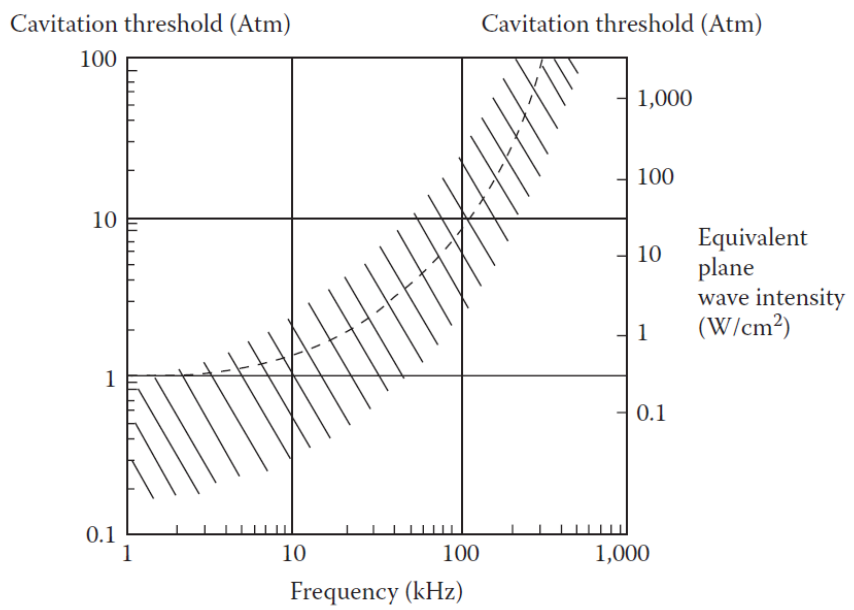

Figure 4. Frequency dependence of the cavitation threshold with continuous wave applied to fresh water at atmospheric pressure. $[1,15]$ ( 2 column fitting image)

The nature of cavitation which is observed can be considered in two categories, stable and transient cavitation. In stable cavitation, a bubble will oscillate with the sound field for several cycles or more of the applied wave field. During this time, the bubble may grow due to rectified diffusion. Rectified diffusion is the growth of a gas bubble driven into oscillation by an appropriate acoustic field [16]. Once the bubble reaches its resonance radius, it will collapse. A larger bubble generally exhibits stable cavitation because it has a lower resonance frequency. In transient cavitation, an oscillating bubble can collapse in one or at most only a few acoustic cycles. However, the distinction between stable and transient cavitation has historically been up to the individual to define, which has caused some confusion. There have been attempts to reconcile these different descriptions, but the basic distinctions have been the bubble life in relation to the period of the applied field and the time it has taken before bubble collapse or the violence of the collapse $[11,12,14]$. In general transient cavitation has tended to cause higher peak pressures and temperatures in the collapsing bubbles. These two phenomena are illustrated in Figure 5 in relation to the compression and rarefaction of an oscillating sound wave.

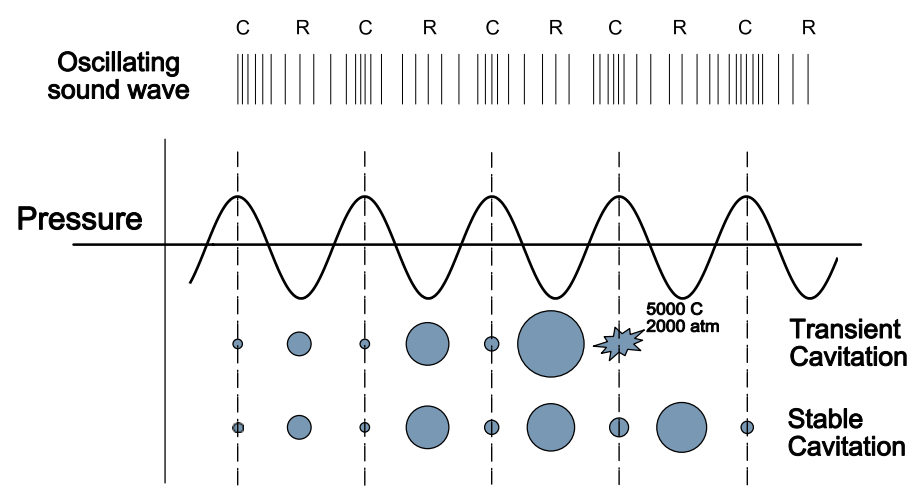

Figure 5. Illustration of stable cavitation bubbles and the lifetime of transient cavitation bubbles in relation to an oscillating sound wave. Adapted from ref $[17,18]$. 


\subsection{Sonomechanical Effects}

A potential target for the use of sonomechanical effects in nuclear process streams is to utilize the increase of agitation and mass diffusion which are the basic phenomena for imparting ultrasonic energy into a process stream. The mechanical effects, are those which occur below the cavitation threshold. In a single phase heterogeneous constituent flow, agitation can be used to enhance mixing. In two-phase flow, such as with inert gas sparging, rectified diffusion is desired to increase the mass transfer and removal rate of dissolved gasses. Using rectified diffusion can be difficult because entrained bubbles tend to avoid sonic fields at lower intensities. However, at higher intensities (those greater than $300 \mathrm{~W} / \mathrm{cm}^{2}$ at $20 \mathrm{kHz}$ ) bubbles no longer avoid the sonic field and begin to break up [19]. Preliminary work, related to the current study, has shown that this phenomenon can significantly increase the overall mass transfer and effectiveness of processes such as inert gas sparging.

\subsection{Fields and Interaction zones}

The ultrasonic interaction zone in front of a high power transducers has a three dimensional structure. For example in the case of the region in front of a horn when transmitting into a semi-infinite liquid there are three distinct zones [1]. These are a "near-horn" zone, a zone with streaming, and then a far-field. The near-horn region, typically within one wavelength from the source is subjected to the highest intensities. Multiple bubbles can be observed forming and collapsing. The streaming zone, typically starting at about one wavelength from the horn, extends out several wavelengths, and it is subjected to lower intensities, commonly those below the cavitation threshold, and its major feature is the streaming field of bubbles, which move out from the near-horn region. The far-field is at some distance where major acoustic streaming has ceased [13]. It is characterized by the lack of macro-streaming effects and energy is mostly dissipated through heating. These phenomena lead to high power ultrasonic reactors that have three classes of fundamental geometry. The first has the horn placed on or very near (within one wavelength) of the surface. The second geometry is where the horn is one wavelength from the surface, or in this case there is also a reflector, which produces a standing wave. The third geometry is where the horn is submerged in a large (effectively a simi-infinite) container where the container surface is far from the horn [1].

All acoustic waves in a fluid have an associated force exerted into a fluid. These forces, when there are bubbles, can act to cause oscillations. If the forces originate from the acoustic source (the horn), they are classified as primary forces or primary Bjerknes forces. The oscillating bubbles in an acoustic field can apply a force on surrounding bubbles. The force due to inter-bubble oscillation are classified as mutual or secondary Bjerknes forces. In general, Bjerkens forces attract bubbles or particles at or below the resonance diameter for the sonic field to an anti-pressure node and those larger that resonance diameter will travel away from those nodes [11]. If the ultrasonic intensity is high enough, these forces can even rip apart bubbles that are in the ultrasonic field. These forces are very difficult to predict or approximate because of their highly nonlinear behavior. Primary Bjerkens forces are easier to predict because they originate from a fixed horn and geometry, but the secondary forces are much more random. Because the secondary forces come from the oscillating bubbles themselves, they can be quite chaotic. The intensity, location, and spatial dimensions of the forces will vary wildly because the sources vary significantly in terms of bubble size and location [11], which also depend on fluid properties including the vapor pressure.

\subsection{Sonochemical effects}

General methods of increasing chemical reactivity include increasing the reaction temperature, concentration of reagent, raising the pressure applied to a system, the use of a catalyst, and irradiation of a system with power ultrasound [14]. Cavitation is the mechanism that makes many sonochemical reactions possible. Cavitation is a violent phenomenon that produces local high temperatures $(\sim 5000 \mathrm{~K}$ or more) and local pressures (up to $\sim 50 \mathrm{MPa})$. This mechanism can provide the temperature and pressure that can be used to overcome the activation energy of a reaction. In addition, cavitation can fragment reagents into reactive radical species, which can catalyze a reaction by providing another pathway for the target reaction to proceed $[7,14]$. This use of acoustic energy to enable sonochemistry can be categorized in four general types of processes: homogeneous sonochemistry of liquids, heterogeneous sonochemistry of liquid-liquid systems, heterogeneous chemistry of liquid-solid systems, and sonocatalysis $[1,20]$.

Although these processes are generally based on cavitation, the enhancement mechanisms are slightly different in their mechanics and applications. In the sonication of homogeneous liquids, the cavitation mechanism applies the cavitation energy to the vapor volume, fragmenting the vapor into highly reactive species. With heterogeneous or immiscible liquids, the agitation of the ultrasound produces emulsions. This produces mixtures with very large surface interaction areas which can result in enhanced reaction rates. With a solid/liquid interface, the enhancement 
mechanism is a combination of physical surface cleaning action and exposing of new solid surfaces. If the solid is in the form of a power or particulate, the cavitation can increase the fragmentation of the particles as well as induce particle surface activation, increasing reaction rates. Sonocatalysis can favor one reaction pathway over another pathway or even provide a reaction pathway that would not be possible conventionally [20].

\section{NOVEL APPLICATIONS OF SONOPROCESSING FOR ADVANCED NUCLEAR TECHNOLOGIES}

There appear to be several potential applications for the use of ultrasonic processing to improve yield for advanced nuclear technologies with applications throughout the nuclear fuel cycle. The nuclear fuel cycle encompasses a series of industrial processes involved in creating the fuel used for generating electricity from nuclear power plants. The cycle starts with uranium ore mining and ends with the final disposition of nuclear waste in a final geologic repository. When fuel is removed from a reactor, the used nuclear fuel (UNF) is held in storage on site and it still contains substantial quantities of fissile material that can potentially be re-processed and materials recycled. In an open fuel cycle, this UNF is ultimately placed into a geologic repository directly. However, in a closed nuclear fuel cycle, this fissile material in UNF can be reprocessed, and the recovered actinides can be reformed in fuel that can be used in power reactors. Reprocessing is typically performed using one or the other of two general processes, known as aqueous and electrochemical processing.

Focusing at the processes employed in the nuclear fuel cycle there are a few steps where ultrasound technologies can potentially be useful. Examples include where ultrasonics is used to reliably remove gasses from liquids and metallic melts [1,21]. Another could be the application of ultrasonics to enhance chemical processes, in much the same way that it is employed in a multitude of industries such as the textile [22], the food [23], and the pharmaceutical industry $[1,4]$.

The nuclear fuel cycle (NFC) is essentially uranium mining, uranium processing, fuel enrichment, fuel fabrication, power production, and spent fuel/waste management. The NFC can either be a closed or open cycle, where the difference is the way that UNF is treated. In an open system, the UNF is stored on site in preparation for final deposition into a final geological repository. In contrast, a closed system will include reprocessing of UNF by chemically or electrochemically separating out unused U-235 and bred Pu-239 for fabrication into mixed oxide (MOX) fuel for electrical production. Currently, the US employs an open NFC and France employs a closed NFC.

\begin{tabular}{|l|l|l|l|}
\hline Uranium Mining & \multicolumn{1}{|c|}{$\begin{array}{c}\text { Uranium } \\
\text { Processing }\end{array}$} & Power Production & $\begin{array}{c}\text { Spent Fuel } \\
\text { Reprocessing }\end{array}$ \\
\hline Uranium Mine & & $\begin{array}{l}\text { In situ purification } \\
\text { system efficiency } \\
\text { enhancement. } \\
\text { enhancement }\end{array}$ & $\begin{array}{l}\text { PUREx process efficiency } \\
\text { improvement and "red oil" } \\
\text { production reduction. }\end{array}$ \\
\hline $\begin{array}{l}\text { Pyro process lanthanide } \\
\text { drawdown process } \\
\text { improvement }\end{array}$ \\
\hline
\end{tabular}

Figure 6. Highlights of the potential applications of ultrasonics in the nuclear fuel cycle (pictures used with permission from the Federation of Electric Power Companies of Japan). (2 column fitting image)

\subsection{Fluoride salt cooled and salt fueled reactor technologies}

Managing tritium release and mitigation in nuclear power reactor systems utilizing molten fluoride salts as a coolant is a very significant issue. There are several examples of such nuclear systems and these include fluoride saltcooled high-temperature reactors (FHRs), molten salt reactors (MSR), and inertial confinement fusion (ICF) reactor concepts. The concern with tritium is its high production rate as well as its permeability through structural materials. In addition, buildup of tritium has potential to negatively impact reactors power generation performance. This tritium production also, particularly in the case of an accidental release, presents a significant hazard to personnel and potentially to the public $[24,25]$.

The most attractive molten fluoride salt under consideration for these technologies is a beryllium-based fluoride salt called flibe, which is a eutectic $2: 1$ by mole mixture of lithium fluoride $(\mathrm{LiF})$ and beryllium fluoride $\left(\mathrm{BeF}_{2}\right)$. This is attractive for its relatively high thermal conductivity and good neutronic performance in comparison to the other 
candidate salts. But, in a neutron flux the salt is a large source of tritium. The mechanisms for the production of tritium are shown in the following nuclear reactions. The $\mathrm{Li}-6$ and $\mathrm{Li}-7$ have a direct path to tritium prodcution, and $\mathrm{Be}-9$ has a more indirect path where it gets transmuted into Li-6 before getting transmuted into tritium [24].

$$
\begin{gathered}
{ }_{3}^{6} \mathrm{Li}+{ }_{0}^{1} \mathrm{n} \rightarrow{ }_{2}^{4} \mathrm{He}+{ }_{1}^{3} \mathrm{H} \\
{ }_{3}^{7} \mathrm{Li}+{ }_{0}^{1} \mathrm{n} \rightarrow{ }_{2}^{4} \mathrm{He}+{ }_{0}^{1} n+{ }_{1}^{3} \mathrm{H} \\
{ }_{4}^{9} \mathrm{Be}+{ }_{0}^{1} n \rightarrow{ }_{2}^{4} \mathrm{He}+{ }_{2}^{6} \mathrm{He} \\
{ }_{2}^{6} \mathrm{He} \rightarrow{ }_{3}^{6} \mathrm{Li}+\beta^{-}+\gamma
\end{gathered}
$$

In all three of the reactor applications, tritium sequestration, tritium containment, and salt purification are important engineering challenges to be addressed in order to demonstrate the viability of these technologies. For instance, in the FHR design the production rate of tritium is significantly larger than current light water reactors (LWR) due to these reactions. On average, a pressurized water reactor (PWR) can produce $1.99 \mathrm{Ci} /$ day [26] where as an FHR would produce $2508 \mathrm{Ci} /$ day [27]. Managing this material is one of the major challenges faced in establishing the viability of the FHR technology $[25,28]$. The molten salt reactor (MSR) shares this issue in addition to the need for fission product gas removal, namely iodine and xenon.

There have been several concepts which have been investigated to address the tritium challenge. First would be the use of tritium "getters" such as graphite or yttrium to capture and immobilize the tritium. Another idea is the use of thin film coatings on heat transfer surfaces. The conditions that favor heat exchange also favor the mass transfer of tritium through barriers. The last idea is the use of gas sparging [28] with mechanical separation. There was a considerable effort put into investigating this technique at Oak Ridge National Laboratory (ORNL) in the Molten Salt Reactor Experiment (MSRE) in the late 1960's and early 1970's. Sparging has been used for removal of fission product gasses, tritium, and the purification of salt [28-31]. This work can be used in designing a tritium removal and salt purification system in advanced salt cooled reactor designs, such as for the FHR.

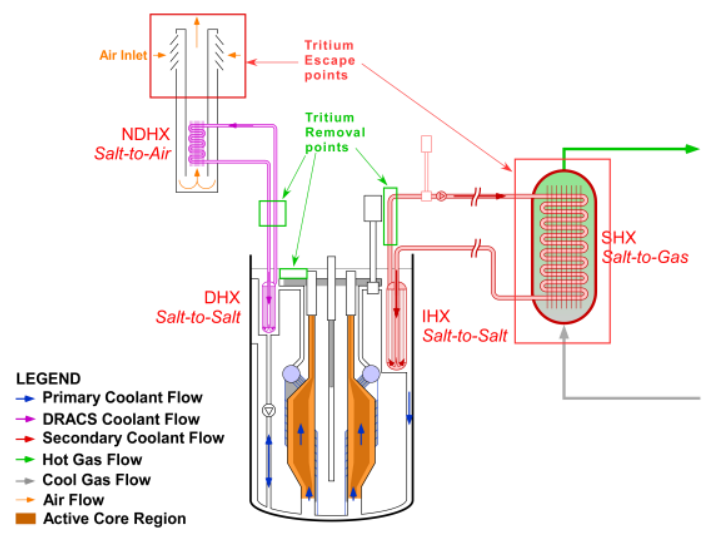

Figure 7. Notional schematic of the of the FHR. Notes the strategic points for tritium removal and the environmental release points. ( 2 column fitting image)

In addition, and slightly related to the tritium challenge, there is the challenge of tritiated hydrofluoric acid (HF/TF) contamination of the flibe. This comes from the presence of hydrogen in the salt as well as production of tritium via any of the paths listed above. This is an issue for both material degradation management and potential personnel safety effects. Solving this challenge can greatly increase the viability of this technology, as well as increase the potential operating life of an FHR plant. 
The more urgent challenge would be to address the production of the large quantity of tritium because it is considered a major design issue which must be solved before the FHR design can be considered technically and economically viable [25]. Inert gas sparging can be used as one possible solution [28], and this process efficiency can be improved by the addition of the use of ultrasonics. The ideal ultrasonic power regime would be in the rectified diffusion regime. This would require that the bubbles be injected at the resonance size for the applied frequency or use the ultrasonic energy to break up larger bubbles into smaller bubbles within the size range needed for resonance. These smaller bubbles would have more mass transfer efficiency due to the larger surface area as well as the agitation effect of the ultrasound. In turn, this would greatly increase the effectiveness of the inert gas sparging technique to sequester dissolved tritium.

Initial investigations have been performed at the University of New Mexico (UNM) to study effects of ultrasound for enhancing the diffusion mass transfer of in-line sparging. A prototypical flow loop was constructed for this study as shown in Fig 8, and preliminary results look to be promising as illustrated by the results shown in Fig 9. The proposed inert gas sparging enhanced by high intensity ultrasonics will cause the stripping bubbles to break into smaller bubbles and increase the convective mass transfer at the liquid gas interface by agitation and increasing gas liquid contact area. The near term goal for this research is to show improvement in sparging using ultrasonics. A set of prototypical ultrasonic flow cells were used to test the theory and some improvement in the degassing was found for a set of given parameters such as gas flow rate, working fluid flow rate, and ultrasonic intensity. Next steps will be to investigate simulant fluids such as glycerol and water mixtures in order to match the key mass transfer nondimensional numbers (Weber, Schmidt, and Sherwood numbers) and look to investigate the scaling arguments for this technology as well as investigate scale-up challenges that will be faced for potential deployment.

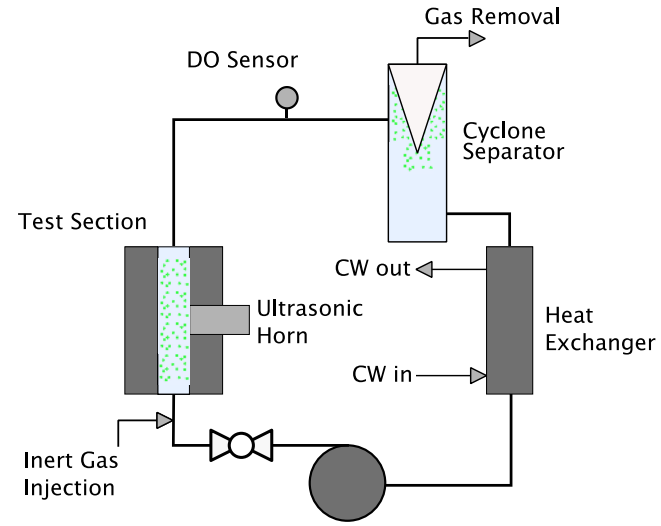

(a)

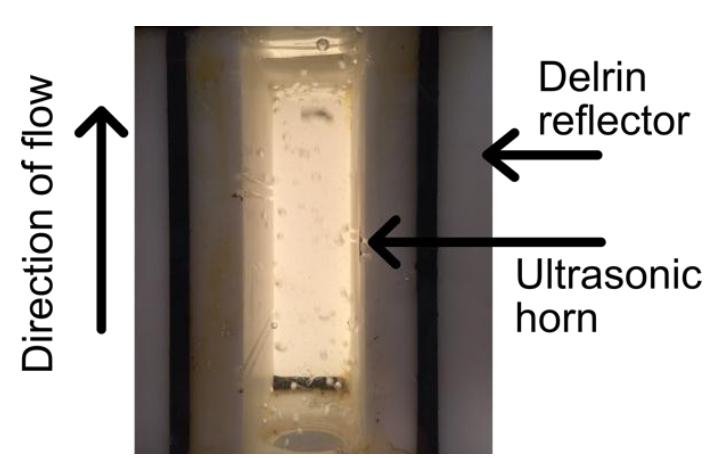

(b)

Figure 8. (a) shows the schematic of the prototype flow cell, and (b) shows the flow through the test section.

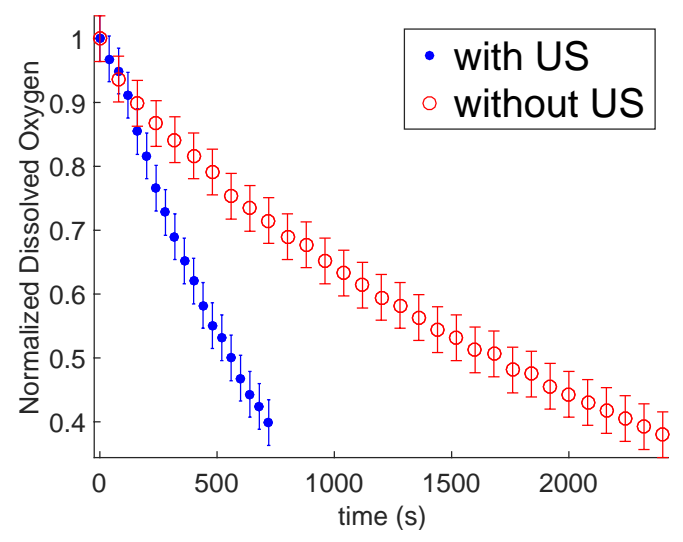

Figure 9. Preliminary results from the prototypical flow cell experiment. This experiment was a prototype small-scale water loop with a volume of 0.8 gallon, inert gas flow of $0.2 \mathrm{sfcm} / \mathrm{min}$, and intensity of $275 \mathrm{~W} / \mathrm{cm}^{2}$. This shows a reduction in oxygen removal time by about $70 \%$. ( 2 column fitting image) 
The preliminary results (shown in Fig 9), indicate that the approach is promising however it is recognized that before there can be an implementation of this technology there are challenges that remain. The challenge of producing small bubbles on the order of $\sim 500 \mu \mathrm{m}$ consistently has been investigated extensively [28]. However, the challenges with using ultrasonics for these types of reactors operating at such high-temperatures and in high radiation fields received very little attention. These issues will have to be investigated to overcoming the effects of tritium migration, high temperatures, potentially high radiation dose, and acoustic coupling. With respect to these challenges, the most advantageous design schemes would appear to be either direct coupling or indirect coupling. This would allow the separation of the ultrasonics from the working fluid as well as enable adding temperature control for the ultrasonic transducer system. It will also provide a barrier to limit the migration of tritium.

The cases of hydrofluoric acid (HF) and tritiated hydrofluoric acid (TF) provide chemical challenges in addition to the radiological challenge. It requires the reduction of ionic tritium/hydrogen into gaseous tritium/hydrogen. This electrochemical process can potentially be coupled with ultrasonics. In order to enhance the reduction of the HF/TF acid to diatomic elemental $\mathrm{H}_{2} / \mathrm{T}_{2}$ and elemental fluorine the ultrasonic intensity must be in the cavitation regime. This can potentially bring several advantages to the electroprocessing cell. The ultrasonic field can sweep away bubbles as they form on an electrode, reduce the ion depletion in the region and assist ion transport, and continuously sonically clean the electrode surfaces [14].

\subsection{Lead Bismuth Eutectic (LBE) cooled reactor technologies}

A lead bismuth eutectic (LBE) has been used as a fast reactor coolant in the past and is being considered as a coolant in advanced reactor concepts as well as for neutron spallation targets and coolant. From previous operational experience, with the Russian nuclear submarine fleet, it is imperative that oxygen concentration be controlled $[32,33]$. Oxygen levels must be maintained within an operational band to prevent corrosion and coolant oxidation. If the oxygen concentration is too low, the passivation layer can detach and accelerate the erosion of the structural material. If the oxygen concentration is too high, the lead in the coolant will oxidize and precipitate causing clogging and obstructions to flow.

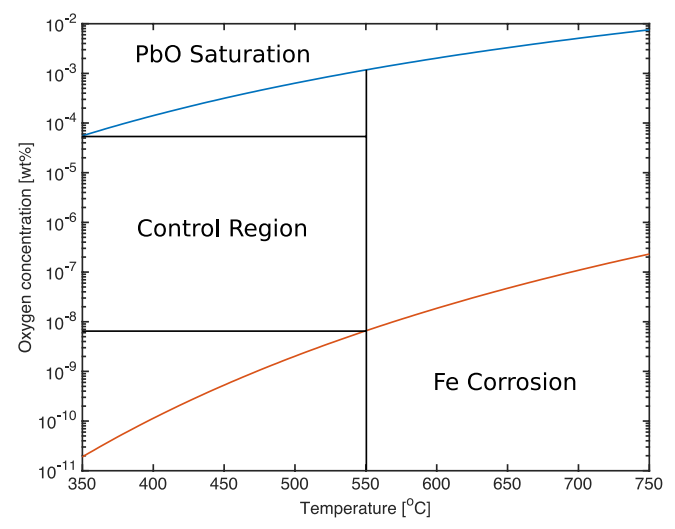

Figure 10. Oxygen Concentration range for operating with LBE [34]. (2 column fitting image)

The control band for a LBE system is shown in Figure 10. Although the band for oxygen control in the figure has boundaries that extend from the precipitation curve (top curve) the corrosion curve (bottom curve), a practical operation range would be more restrictive to provide an operational margin of error. This is a challenge due to the kinetics of oxygen control. There are two methods to lower the oxygen concentration. The first and more conventional method uses hydrogen gas bubbling. This produces water vapor, which can then be removed at any free surface. However, this process is quite slow and depends on the concentration of hydrogen used. The other method of oxygen removal is using a cold trap. This works by lowering the temperature of the LBE, trapping oxygen in the form of lead oxide $(\mathrm{PbO})$ where it precipitates and it can then be removed by mechanical filtration.

In considering options, the method of increasing oxygen is difficult because of the speed at which $\mathrm{Pb}$ oxidizes into $\mathrm{PbO}$. This is quite a fast reaction, so oxygen bubbling is not feasible due to the speed at which the injection site will clog $[34,35]$. Therefore, methods for increasing controlled oxygen concentration have been investigated. One of 
the methods that has been investigated is the use of in-line $\mathrm{PbO}$ spheres designed to increase the oxygen concentration in a more controlled manner [33].

The cleaning of a fluid using hydrogen bubbling can take significant time. In a typical system with current designs this can be on the order of a day to lower oxygen concentration from $10^{-5} \% w t$ to $10^{-6} \% w t$. The limitations on the reaction rate can come from a couple of factors. First consideration would be the hydrogen concentration in the cleaning gas. Using pure hydrogen has some inherent safety considerations, so a cleaning gas would contain some inert carrier gas. For example, a mix of $6 \%$ hydrogen with the balance Argon (Ar) is used on at experimental facilities such as the Development of Lead Alloy Technical Applications (DELTA) Loop at Los Alamos National Laboratory (LANL) [35]. Because the reaction is so slow, the hydrogen may pass through the system and not react before it will leave through a free surface.

Ultrasonics has been successfully used in the treatment of light alloy melts [2], so the same principles and techniques can potentially apply when used with LBE. The gas usage efficiency can theoretically be increased using high-powered ultrasonics by increasing the reduction reaction rate of $\mathrm{PbO}$ due to sonochemical effects. In addition, through the sonomechanical effects, dissolved oxygen can be coalesced and liberated at free surfaces. Through the use of high-powered ultrasonics, the LBE cleaning time can potentially be reduced through sonochemistry effects by either increasing the reaction rate or simply by removing the oxygen from solution. With some effort, a sonochemical flow cell, like the one shown in Fig. 1, could be designed for use in this application to reduce PbO in solution. Also, in order to take advantage of the degassing effects of ultrasonics, tanks with free surfaces can be fitted with horns or transducers to degas dissolved oxygen into the free surface more quickly. This can either be used in any expansion or settling tanks as well as a specifically designed tank for oxygen removal. This can potentially be implemented using established liquid metal degassing techniques.

\subsection{Spent Nuclear Fuel Separation Technologies}

The back end of the nuclear fuel cycle (fuel after use in a reactor) in most countries consists of a final geological repository for UNF which will house the material, essentially forever. From a resource utilization perspective this is not an efficient use of unused actinides in the nuclear fuel. The current LWR fleet in an open fuel cycle leaves a significant amount of fissile material in the spent nuclear fuel [36]. To close the fuel cycle and reprocess, or not, has become a political rather than a technical one. In some countries, there is an incentive to reprocess the UNF to enable utilization of this unused fissionable material. Reprocessing spent fuel will also reduce the quantities of long lived radioactive isotopes that will remain in the final waste form.

There are currently two main methods of reprocessing UNF. The first is the PUREX (Plutonium Uranium Redox Extraction) process and is an aqueous chemical process to separate $\mathrm{Pu}$ and $\mathrm{U}$. This has to date been the standard method for reprocessing. The second method is pyroprocessing which is an electrochemical process that utilizes high temperature molten salts or metals for separating fissile material from UNF [37]. Ultrasonics appears to have the potential to enhance aspects of both of these processes [6].

\subsubsection{Aqueous Separation using Sonoprocessing Methods (PUREX)}

The average lifetime of nuclear fuel in the reactor is approximately three years with one-third of the fuel being removed each year, although there have been recent attempts looking at extending fuel lifetimes [38]. Chemically, the U-235 and U-238 component in the fuel will be reduced to $1 \mathrm{wt} \%$ and $94 \mathrm{wt} \%$, respectively. When removed from the core the used fuel will be stored for about 150 days and reprocessing can then be considered using the PUREX process.

Detailed descriptions of the PUREX process can be found in literature $[38,39]$. It involves a dissolution step where pieces of fuel are dissolved in near boiling $10 \mathrm{M} \mathrm{HNO}_{3}$. This step has been proven to be effective, but it can take several hours to dissolve the uranium, plutonium and fission products. Once the uranium and plutonium are dissolved, a solvent, tri-butyl phosphate (TBP), is added to the solution which forms a complex around the uranium and plutonium compounds that allows the uranium and plutonium to be easily separated.

$$
\begin{gathered}
\mathrm{UO}_{2}^{+2}+2 \mathrm{NO}_{3}^{-}+2 \mathrm{TBP} \leftrightharpoons U \mathrm{U}_{2}\left(\mathrm{NO}_{3}\right)_{2} \cdot 2 \mathrm{TBP} \\
\mathrm{Pu}^{+4}+4 \mathrm{NO}_{3}^{-}+2 \mathrm{TBP} \leftrightharpoons \mathrm{Su}\left(\mathrm{NO}_{3}\right)_{4} \cdot 2 \mathrm{TBP}
\end{gathered}
$$

The length of the dissolving time and the high concentration of $\mathrm{HNO}_{3}$ employed provide a strong motivation to find an innovative way of improving the efficiency of the process, potentially enabling a reduction in the volume or concentration of $\mathrm{HNO}_{3}$. A sonoprocessing method could potentially be used to enhance the chemical process by first exploring the mockup experimental design to provide a proof-of-concept and then later conduct experiments with 
surrogate depleted uranium oxide. Successful data may help identify a processing path that can reduce nuclear power plant operation cost, processing time, and increase the fuel conversion efficiency.

\subsubsection{Pyrochemical Reprocessing (Pyroprocessing)}

Pyroprocessing is a method to reprocess and recycle UNF. When fuel is depleted within a reactor, the final composition of the fuel is roughly $95 \%$ uranium, $1 \%$ transuranics, and $4 \%$ unusable fission products [40]. Through this process, the remaining fissionable material in the UNF can be separated out, concentrated, and refabricated into new nuclear fuel that can then be used in a fast spectrum advanced reactor. In order to do this, the pyroprocess utilizes an electrorefiner (ER) in a molten $\mathrm{LiF}$ based salt. The basic operation of the electrorefiner uses a redox reaction driven by an electrical bias. The UNF is placed into an anode basket inside of a $\mathrm{LiCl}-\mathrm{KCl}-\mathrm{UCl}_{3}[41]$ molten salt, and because of the electrical bias, the uranium will plate out a solid cathode and some uranium, rare earths, and transuranics will migrate to a liquid cadmium cathode. With extended use, rare earth contamination and fission product contamination accumulates in the refining salt, increasing the heat production due to radioactive decay driving the need for the salt to be replaced or purified [40].

Because of the cost of production and disposal of the salts, there is an incentive to be able to purify the salt and remove fission products. One method to do this, which that is actively being investigated, is oxide precipitation, with the use of an additional oxidant, such as $\mathrm{Li}_{2} \mathrm{O}$ or $\mathrm{V}_{2} \mathrm{O}_{5}$, phosphate precipitation, or carbonate precipitation [42].

Technology for the pyrochemical treatment of UNF has been under active development at Argonne National Laboratory (ANL) and Idaho National Laboratory (INL) to treat UNF from the Experimental Breeder Reactor-II (EBR-II) and possibly from Light Water Reactors (LWRs) in the future [41].

The Korea Atomic Energy Research Institute (KAERI) has been the leading organization engaged in work on the eutectic salt waste treatment process. Currently, rare-earth elements resulting from the electrorefining process are being converted to their molten salt as insoluble precipitates by a reaction with oxygen in the oxidative precipitation step. These results in their oxychloride and oxide forms [42]. Under the current studied conditions, in a typical reactor, it would have to operate at a $750{ }^{\circ} \mathrm{C}$ salt temperature with oxygen sparging time, and rate of more than 7 hours and 5 $\mathrm{L} / \mathrm{min}$, respectively, to achieve $99 \%$ oxidation efficiency. It was shown that operating the system at $800{ }^{\circ} \mathrm{C}$ would reduce the sparging time to 6 hours [42].

The use of sonoprocessing may help improve process performance by increasing the oxidation efficiency and decreasing the sparging time. Komarov provides a literature review on high power ultrasonics in pyrometallurgy [43], which validated that sonoprocessing may be used in electrochemical reprocessing of UNF, especially during the oxidative precipitation in order to enhance its overall performance.

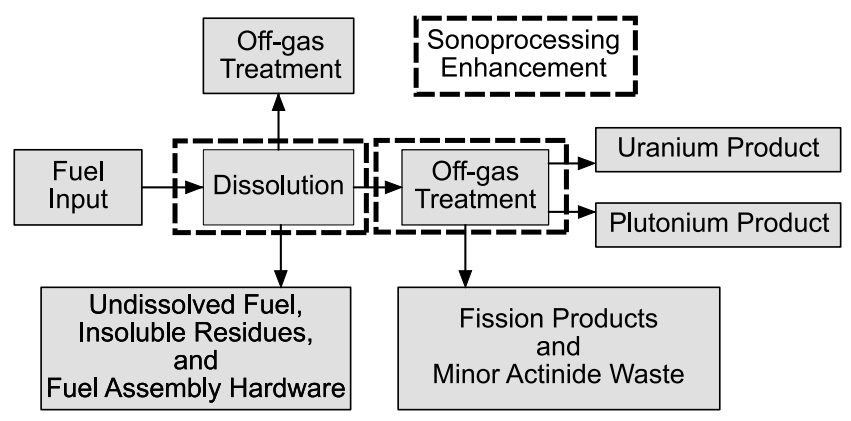

Figure 11. PUREX flow path illustrating the potential unit operations for sonoprocessing enhancement with ultrasonic techniques designated by the dashed boxes. 


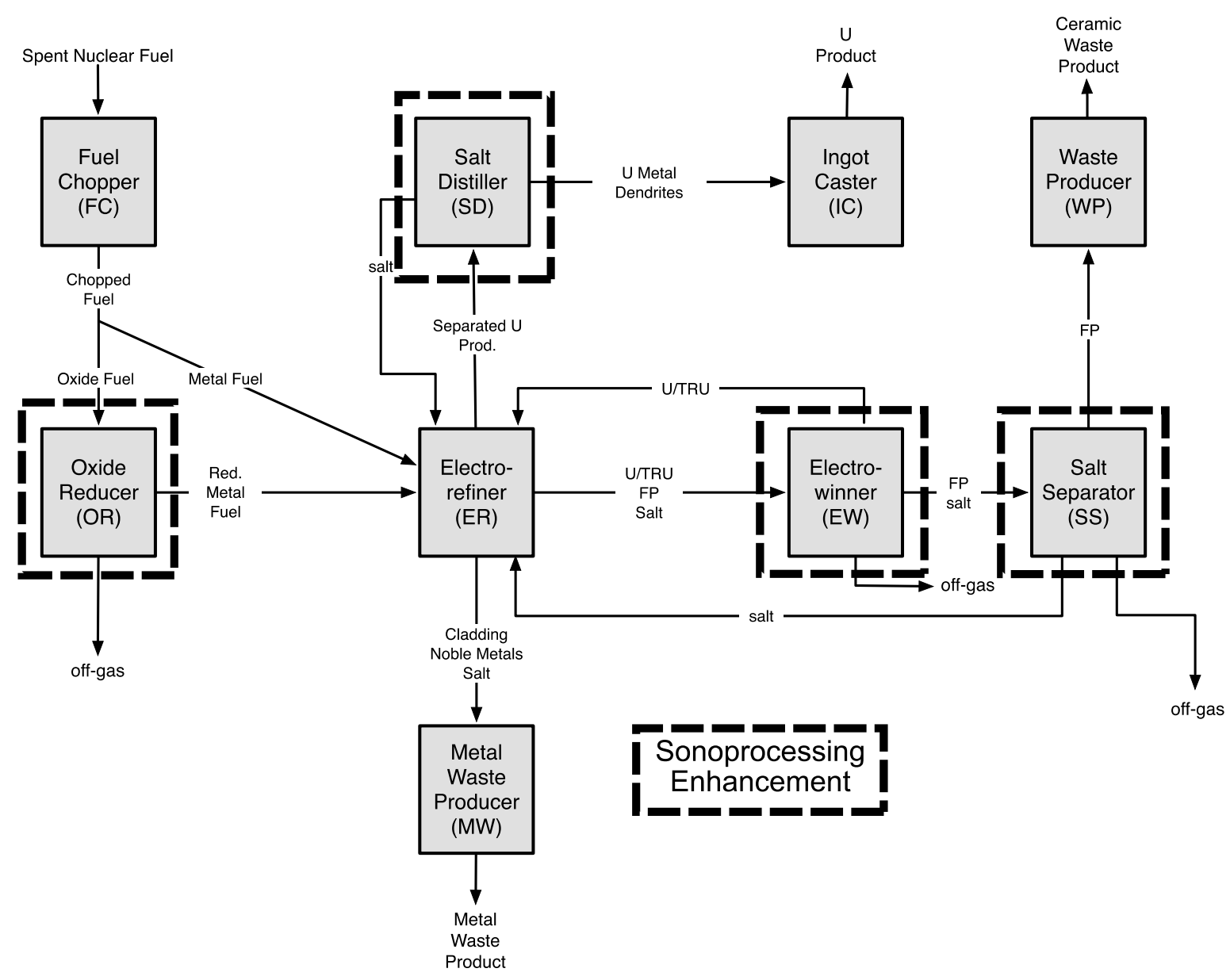

Figure 12. Pyroprocessing flow path illustrating potential unit operations for sonoprocessing enhancement with ultrasonic techniques designated by the dashed boxes. ( 2 column fitting image)

\section{CHALLENGES WITH INTEGRATING ULTRASONIC TECHNOLOGY FOR NUCLEAR PROCESS STREAMS}

Applying ultrasonics to nuclear process streams presents unique challenges which would require careful consideration and focused effort to resolve. The harsh conditions that come with nuclear applications include ensuring use of materials that are compatible with advanced reactor coolants and high and low $\mathrm{pH}$ process fluid streams, radiation resistance, including for high power ultrasound transducers, and ensuring the ability to function at high temperatures. Each challenge can be potentially solved individually, but the larger challenge is to devise a solution that will satisfy all challenges in concert. In the following section, each challenge is considered in the context of sonoprocessing for nuclear process applications.

\subsection{Materials compatibility challenges}

The materials challenges with ultrasonics is primarily dictated by the application and sonic intensities used. In general, expected erosion/wear due to localized cavitation and horn or transducer material compatibility are common design criteria for both direct and indirect applications of ultrasonics. These process cells will be in contact with corrosive fluids, high temperatures, and significant radiation fields. These material challenges have been considered extensively and using past experience they can be resolved by careful material selection in conjunction with the application of direct and indirect sonication techniques. Direct immersion applications will clearly have to use horns and transducers that will be chemically compatible with the working fluid. Indirect sonication techniques have a clear advantage in this area, but with the tradeoff of having to use higher ultrasonic intensities. 
In nuclear reactor applications or any application where there is a substantial neutron flux, wear products can be an important consideration. In addition, some proposed coolants are quite corrosive and operate at much higher temperatures than conventional reactors. In spent nuclear fuel processing, activation of wear products would be less of a concern, but chemical compatibility is more of a concern. These factors should be taken into consideration when choosing materials as well as choosing between direct and indirect sonication techniques.

In addition to the materials in contact, it is necessary to consider the temperature and radiation resistance of the transducer materials. For example if a piezoelectric transducer is used operating temperatures need to be below the Curie temperature and selection needs to consider response to both gamma and neutrons [44-47]. The magneto restrictive family of transducers may provide attractive candidates with both good thermal resistance and radiation resistance properties. There has been some work that addresses the use of these in advanced reactor applications [47], but there is still much to be investigated in this area.

\subsubsection{Materials Challenges for Immersion Type Applications}

There is a significant literature that considers the compatibility between horns and transducers with various processing fluids together with significant operational history [1]. For example, there has been success in the use of liquid sonoprocessing cells for the chemical industry and molten metal degassing. Examples of current off the shelf transducers and horns exist that are compatible with aqueous fluids and others can be used in molten metal applications. These horns are commonly made using an alloy of titanium or aluminum [48]. However, to utilize the technologies and techniques for a nuclear process application, there are a few more considerations to resolve beyond chemical resiliency. If the application utilizes an intensity that would result in wear and cavitational erosion of the horn, the wear products must be benign in the process stream as well as not become appreciably activated in a neutron flux. Erosion product contamination of a process stream has been investigated in the food production industry where contamination prevention is a priority [23]. Another approach is to also to seek to minimize erosion through the use of designs such as a ring transducer geometry [1]. This being said, direct immersion would be a good candidate for use in spent fuel reprocessing because the neutron fluxes are smaller and the initial contamination levels are much higher. In this case, the added contamination of horn cavitational erosion/wear products would be negligible from a contamination point of view.

\subsubsection{Materials Challenges for Indirect Sonication Applications}

In contrast to direct immersion applications, indirect application of ultrasonics can provide a more separated technique, which can provide more flexibility for designs and applications. However, other challenges arise depending on how the ultrasonics will be coupled to the system. For systems where the horn or transducer is directly coupled to the wall of the sonochemical/sonomechanical reactor, the design of the coupling interface would have to be considered. Generally, these couplings are achieved using either a mechanical loading (clamping), an epoxy or high temperature solder compounds. Use of any organic compound in a radiation flux has issues due to material degradation. Also there are mechanical design issues: for example, if the application has a large change in temperature, materials with similar thermal expansion coefficients must be used as well as a coupling adhesive appropriate for the temperature ranges. However, if there is an appreciable difference in temperature, a direct coupling may prove impractical. In this case, the use of a coupling fluid jacket as shown in Fig. 2 will be appropriate. The material challenges can potentially be solved with more flexibility in terms of coupling fluids and temperature ranges. However, the use of an intermediate fluid, the acoustic efficiency will be lower. This leads to having to increase applied acoustic power for the desired acoustic power delivered, and subsequently, the possible increased cavitational erosion of the ultrasonic horn and structural materials.

The technique of indirect sonication has been successfully used in many ultrasonic cleaning bath applications. It has also been implemented in flowing process streams, and it has been shown to be effective in a flow-through lysis module for the disruption of bacterial spores by Warner et al [49]. What is yet to be demonstrated is the long-term reliability and equipment lifetimes for continuously running systems.

\subsection{High Radiation Dose}

Common to all nuclear applications, radiation damage to ultrasonic components is an important design consideration. The two major types of damage are damage from gamma and neutron radiation. Both types of damage have different damage and failure mechanisms because of differences in the way different radiation interacts with matter. Generally, gamma damage is less of a concern because most piezoelectrics can function with significant dose rates [50]. 
Considering radiation damage to the transducer, the individual components of the transducer must be addressed separately. First of which is the piezoelectric element. From operation experience and observation is is assumed that gamma radiation damage is mostly dependant on total accumulated dose, not the dose rate, granted that there is no significant heating of the material from the high dose rate [50]. There are three basic effects on piezoelectric performance. As dose increases, the first and primary mechanism is depoling. In terms of neutron radiation damage to transducers, some studies showed prolonged exposure to neutron radiation did not cause catastrophic failure, but a significant drop in their sensitivity [51]. Also, there is a drop in efficiency over time and frequent recalibrations and replacement is necessary [52]. A review report and investigation of in-core ultrasonic sensors from INL assessed the candidate transducer materials and came up with two candidate materials, bismuth titanate and aluminum nitride for their currently known material properties, notably the high Curie temperature and gamma and neutron resiliency [44,47]. In addition to looking at piezoelectric transducers, the INL investigation looked into magnetorestrictive transducers. This study had identified four candidate mangetostrictive materials: Remendur, Galfenol, Arnakrome 4, and Arnakrome 5 [44,45].

Gamma radiation can also affect the structural components of a transducer. There are organically based components that are used in transducers such as plastics, adhesives, and epoxies. These organically based components tend to be the weak point of the assembly. There are known adhesives and epoxies that have shown good performance [50]. Therefore, it is important that the material selection should be tailored to the anticipated radiation field. The housing, usually made of stainless steel, would be resilient to the gamma radiation. Therefore, for the anticipated gamma field, most housings will be adequate. The cabling is also another concern, but there are also known cabling materials that have been radiation hardened used in high dose environments [50].

The information on radiation damage versus reliability of transducers, particularly for high power applications, is far from comprehensive and more study into this topic is necessary. Currently, there are various projects in the international ultrasonic NDT community that are investigating acoustic in-core sensors [47]. One example of a high radiation flux and high temperature sensor is the REMORA 3 experiment, which is designing an online gas composition measurement sensor that works via acoustic sensor inside of the internal rod plenum [53]. Another project had recently irradiated single crystal aluminum nitride transducers in the MIT test reactor with which shows favorable performance of aluminum nitride in an in-core radiation field [54]. Due to the two-way nature of this technology, this sensor technology can be repurposed into power ultrasonics with some modifications. There are also several projects looking at the challenges of in-sodium operation for fast reactors to provide under sodium viewing [45,55].

\subsection{High temperature}

Many of the high temperature applications of ultrasonics have been investigated in situations where there are high radiation fluxes [50]. There is a comprehensive review article for high temperature transducers by Kažys [52] which considers the state of the art and highlights key issues. The anticipated application where this would immediately find application is transducers for NDT and structural health monitoring in current and advanced nuclear power reactors. Various experimental reactors and advanced reactor designs are also looking for ultrasonic transducer designs for various in-core applications, including fuel evolution during initial loading and bring a reactor to power. Various candidate materials [39] for both piezoelectric and magnetostrictive materials have been considered for high temperature applications. However, there is still limited experience on which to draw conclusions regarding transducers or horns used in prolonged immersion and operation in liquid metals or molten salts. There is published data on the combination of high temperatures (>600 C) and high gamma fields (>30KGy/h) consolidated in ref [50]. There is still much room for more data and investigation in high temperature transducers.

\section{SUMMARY}

The application of high power ultrasonics for sonoprocessing is a technology whose potential has not been fully realized or quantified. The science of high power ultrasonics is still difficult to quantify because of its inherent nonlinear behavior. Despite this, it has had industrial and economic successes in many non-nuclear applications, but with that being said, the full potential of this technology has not been tapped. This field has many possible applications which are being investigated, but those within the nuclear fuel cycle have received only limited attention. Because of this, the field of nuclear application of high powered ultrasonics is very open and full of possibilities.

For the FHR, the tritium challenge is being investigated by enhancing the inert gas sparging with ultrasonic processing. There is still much work required to demonstrate the viability of this approach in the context of other methods of tritium release and mitigation that are currently being investigated. 
The second technology challenge addressed was the oxygen control in LBE systems. This technology could potentially be enhanced by applying high power ultrasonics to speed up the reduction of $\mathrm{PbO}$ for cleaning as well as facilitate the controlled addition of oxygen in LBE.

UNF reprocessing also can be enhanced by utilizing the sonochemical and sonomechanical behavior to increase the efficiency of reprocessing by lowering the time required for the chemical processes. In order to deploy these enhancements, there are still many things to investigate, most notably would be the long term survivability of the ultrasonic technology in the high temperature, high radiation, and chemically hostile nuclear environment.

Currently, there is work showing that ultrasonics can be placed in these operating conditions for NDT/NDE purposes, but because of the reversibility of ultrasonic technologies, this effort can be easily adapted for power ultrasonics and processing. Ultimately, the deciding factor in terms of potential applications would be the economic viability of deploying this technology. Since the nuclear industry has such high capital costs, it is probable that high power ultrasonic technologies will be an attractive and indispensable addition to many nuclear processes.

\section{ACKNOWLEDGEMENTS}

This work is supported by a Department of Energy Nuclear Energy University Program (NEUP) Integrated Research Project (NEUP14-7476). Leonard Bond is supported through the Center for NDE at Iowa State University, a recently (January 2016) graduated NSF IU CRC research center. The authors would like to thank Cole Mueller for his invaluable contributions to this project. They would also like to thank Bruce Green from Sonics and Materials for his helpful discussions and technical assistance. 


\section{REFERENCES}

[1] D. Ensminger, L. Bond, Ultrasonics, 3rd ed., CRC Press, New York, 2011.

[2] G.I. Eskin, D.G. Eskin, Ultrasonic Treatment of Light Alloy Melts, Second Edition, CRC Press, 2014.

[3] K.S. Suslick, G.J. Price, Applications of Ultrasound to Materials Chemistry, Annu. Rev. Mater. Sci. 29 (1999) 295-326. doi:10.1146/annurev.matsci.29.1.295.

[4] M. Sivakumar, S.Y. Tang, K.W. Tan, Cavitation technology - a greener processing technique for the generation of pharmaceutical nanoemulsions., Ultrason. Sonochem. 21 (2014) 2069-83. doi:10.1016/j.ultsonch.2014.03.025.

[5] O. Mitsuru, K. Yutaka, K. Masahiro, T. Masahiro, N. Yoshiaki, Advanced inspection technologies for nuclear power plants, Hitachi Rev. 58 (2009) 82-87.

[6] S.I. Nikitenko, L. Venault, R. Pflieger, T. Chave, I. Bisel, P. Moisy, Potential applications of sonochemistry in spent nuclear fuel reprocessing: A short review, Ultrason. Sonochem. 17 (2010) 1033-1040. doi:10.1016/j.ultsonch.2009.11.012.

[7] L.H. Thompson, L.K. Doraiswamy, Sonochemistry: Science and Engineering, Ind. Eng. Chem. Res. 38 (1999) 1215-1249. doi:10.1021/ie9804172.

[8] N.P. Vichare, P. Senthilkumar, V.S. Moholkar, P.R. Gogate, A.B. Pandit, Energy Analysis in Acoustic Cavitation, Ind. Eng. Chem. Res. 39 (2000) 1480-1486. doi:10.1021/ie9906159.

[9] Petrosonic Announces Successful Completion of Engineering Feasibility Analysis - MarketWatch, (n.d.). http://www.marketwatch.com/story/petrosonic-announces-successful-completion-of-engineering-feasibilityanalysis-2014-06-10 (accessed July 6, 2015).

[10] G. Harvey, A. Gachagan, T. Mutasa, Review of high-power ultrasound-industrial applications and measurement methods., IEEE Trans. Ultrason. Ferroelectr. Freq. Control. 61 (2014) 481-95. doi:10.1109/TUFFC.2014.2932.

[11] T.G. Leighton, The Acoustic Bubble, Academica Press, London, 1994.

[12] R.E. Apfel, Acoustic cavitation prediction, J. Acoust. Soc. Am. 69 (1981) 1624-1633. doi:10.1121/1.385939.

[13] R.T. Beyer, Nonlinear Acoustics, Acoustical Society of America, 1997.

[14] T.J. Mason, J.P. Lorimer, Sonochemistry: Theory, Applications and Uses of Ultrasound in Chemistry, John Wiley \& Sons, New York, 1988.

[15] R.J. Urick, Principles Of Underwater Sound, McGraw-Hill Ryerson, Limited, 1983.

[16] P.R. Birkin, T.G. Leighton, Y.E. Watson, The use of acoustoelectrochemistry to investigate rectified diffusion., Ultrason. Sonochem. 11 (2004) 217-21. doi:10.1016/j.ultsonch.2004.01.023.

[17] J.-L. Capelo-Martnez, ed., Ultrasound in Chemistry, Wiley-VCH Verlag GmbH \& Co. KGaA, Weinheim, Germany, 2008. doi:10.1002/9783527623501.

[18] T. Leong, M. Ashokkumar, S. Kentish, The fundamentals of power ultrasound - a review, Acoust. Aust. 39 (2011) 54-63.

[19] T.K. Jagannathan, R. Nagarajan, K. Ramamurthi, Effect of ultrasound on bubble breakup within the mixing chamber of an effervescent atomizer, Chem. Eng. Process. Process Intensif. 50 (2011) 305-315.

doi:10.1016/j.cep.2011.01.006. 
[20] T.J. Mason, ed., Chemistry with Ultrasound, Elsevier Applied Science, New York, NY, 1990.

[21] T.T. Meek, Q. Han, H. Xu, Degassing of Aluminum Alloys Using Ultrasonics Vibration, Oak Ridge, TN, 2006.

[22] T. Harifi, M. Montazer, A review on textile sonoprocessing: A special focus on sonosynthesis of nanomaterials on textile substrates, Ultrason. Sonochem. 23 (2015) 1-10. doi:10.1016/j.ultsonch.2014.08.022.

[23] R. Mawson, M. Rout, G. Ripoll, P. Swiergon, T. Singh, K. Knoerzer, et al., Production of particulates from transducer erosion: implications on food safety., Ultrason. Sonochem. 21 (2014) 2122-30. doi:10.1016/j.ultsonch.2014.04.005.

[24] J. Serp, M. Allibert, O. Beneš, S. Delpech, O. Feynberg, V. Ghetta, et al., The molten salt reactor (MSR) in generation IV: Overview and perspectives, Prog. Nucl. Energy. 77 (2014) 308-319. doi:10.1016/j.pnucene.2014.02.014.

[25] V.K. Varma, D.E. Holcomb, F.J. Peretz, E.C. Bradley, D. Ilas, A.L. Qualls, et al., AHTR Mechanical, Structural, And Neutronic Preconceptual Design, Oak Ridge, TN (United States), 2012. doi:10.2172/1081980.

[26] NRC: Frequently Asked Questions About Liquid Radioactive Releases, (n.d.). http://www.nrc.gov/reactors/operating/ops-experience/tritium/faqs.html\#normal (accessed December 31, 2015).

[27] Fluoride-Salt-Cooled High Temperature Reactor (FHR) Materials, Fuels and Components White Paper, Madison, Wisconsin, 2013.

[28] T.S. Kress, Mass transfer between small bubbles and liquids in concurrent turbulent pipeline flow, University of Tennessee, 1972.

[29] M.W. Rosenthal, P.R. Kasten, R.B. Briggs, Molten-Salt Reactors-History, Status, and Potential, Nucl. Technol. 8 (1970) 107-117.

[30] C.H. Gabbard, Development of a venturi type bubble generator for use in the molten-salt reactor xenon removal system, ORNL-TM-4122, Oakridge, TN, 1972.

[31] R.B. Lindauer, MSRE Design and Operations Report Part VII Fuel Handling and Processing Plant, Oak Ridge, TN, 1967.

[32] M. Caro, K. Woloshun, F. Rubio, S.A. Maloy, P. Hosemann, Heavy liquid metal corrosion of structural materials in advanced nuclear systems, JOM. 65 (2013) 1057-1066. doi:10.1007/s11837-013-0663-7.

[33] A. Marino, J. Lim, S. Keijers, J. Van Den Bosch, J. Deconinck, F. Rubio, et al., Temperature dependence of dissolution rate of a lead oxide mass exchanger in lead-bismuth eutectic, J. Nucl. Mater. 450 (2014) 270277. doi:10.1016/j.jnucmat.2013.12.023.

[34] J. Zhang, N. Li, Y. Chen, Oxygen control technique in molten lead and lead-bismuth eutectic systems, Nucl. Sci. Eng. 154 (2006) 223-232.

[35] N. Li, T. Darling, Oxygen Control Methodology and Calibration Strategy, Los Alamos, 2002.

[36] Processing of Used Nuclear Fuel - World Nuclear Association, (n.d.). http://www.worldnuclear.org/information-library/nuclear-fuel-cycle/fuel-recycling/processing-of-used-nuclear-fuel.aspx (accessed April 26, 2016). 
[38] M. Benedict, T.H. Pigford, H.W. Levi, Nuclear Chemical Engineering, McGraw-Hill, 1981.

[39] R.G. Wymer, B.L. Vondra, Light water reactor nuclear fuel cycle, CRC Press, 1981.

[40] S. Phongikaroon, R.W. Bezzant, M.F. Simpson, Measurements and analysis of oxygen bubble distributions in LiCl-KCl molten salt, Chem. Eng. Res. Des. 91 (2013) 418-425. doi:10.1016/j.cherd.2012.09.010.

[41] J.J. Laidler, J.E. Battles, W.E. Miller, J.P. Ackerman, E.L. Carls, Development of pyroprocessing technology, Prog. Nucl. Energy. 31 (1997) 131-140. doi:10.1016/0149-1970(96)00007-8.

[42] Y.-Z. Cho, T.-K. Lee, J.-H. Choi, H.-C. Eun, H.-S. Park, G.-I. Park, Eutectic(LiCl-KCl) Waste Salt Treatment by Sequencial Separation Process, Nucl. Eng. Technol. 45 (2013) 675-682.

doi:10.5516/NET.06.2013.022.

[43] S. V. KOMAROV, M. KUWABARA, O. V. ABRAMOV, High Power Ultrasonics in Pyrometallurgy: Current Status and Recent Development, ISIJ Int. 45 (2005) 1765-1782.

doi:10.2355/isijinternational.45.1765.

[44] J. Daw, J. Rempe, J.P. Inl, P. Ramuhalli, R.M. Pnnl, H.T.C. Anl, NEET In-Pile Ultrasonic Sensor Enablement-FY-2013 Status Report, (2013).

[45] P.N. Bilgunde, L.J. Bond, Effect of Thermal Degradation on High Temperature Ultrasonic Transducer Performance in Small Modular Reactors, Phys. Procedia. 70 (2015) 433-436.

doi:10.1016/j.phpro.2015.08.137.

[46] J.W. Griffin, G.J. Posakony, R. V. Harris, D.L. Baldwin, A.M. Jones, L.J. Bond, High temperature ultrasonic transducers for in-service inspection of liquid metal fast reactors, in: 2011 IEEE Int. Ultrason. Symp., IEEE, 2011: pp. 1924-1927. doi:10.1109/ULTSYM.2011.0479.

[47] J. Rempe, H. MacLean, R. Scheley, D. Hurley, J. Daw, S. Taylor, et al., New In-Pile Instrumentation to Support Fuel Cycle, (2011).

[48] Branson Ultrasnoinc Horn Catalog, (n.d.). http://www.emersonindustrial.com/enUS/documentcenter/BransonUltrasonics/Plastic Joining/Ultrasonics/Products/Branson_Horn_Catalog.pdf (accessed December 31, 2015).

[49] C.L. Warner, C.J. Bruckner-Lea, J.W. Grate, T. Straub, G.J. Posakony, N. Valentine, et al., A Flow-Through Ultrasonic Lysis Module for the Disruption of Bacterial Spores, J. Assoc. Lab. Autom. 14 (2009) 277-284. doi:10.1016/j.jala.2009.04.007.

[50] A.N. Sinclair, A.M. Chertov, Radiation endurance of piezoelectric ultrasonic transducers--a review., Ultrasonics. 57 (2015) 1-10. doi:10.1016/j.ultras.2014.10.024.

[51] M.R. Severson, An Experimental Design for Measuring In Situ Radiation Damage to a Piezoelectric Transducer, Wright-Patterson Air Force Base, 2004.

[52] R. Kažys, A. Voleišis, B. Voleišienè, High temperature ultrasonic transducers: review, Ultragarsas (Ultrasound). 63 (2008) 7-17.

[53] T. Lambert, E. Muller, E. Federici, E. Rosenkrantz, J.Y. Ferrandis, X. Tiratay, et al., REMORA 3: The first instrumented fuel experiment with on-line gas composition measurement by acoustic sensor, in: 2011 2nd Int. Conf. Adv. Nucl. Instrumentation, Meas. Methods Their Appl., IEEE, 2011: pp. 1-6. doi:10.1109/ANIMMA.2011.6172924.

[54] B. Reinhard, B.R. Tittmann, A. Suprock, Nuclear Radiation Tolerance of Single Crystal Aluminum Nitride Ultrasonic Transducer, Phys. Procedia. 70 (2015) 609-613. doi:10.1016/j.phpro.2015.08.036.

[55] J.W. Griffin, L.J. Bond, T.J. Peters, K.M. Denslow, G.J. Posakony, S.H. Sheen, et al., Under-Sodium 
Viewing: A Review of Ultrasonic Imaging Technology for Liquid Metal Fast Reactors, 2009. 